\title{
A Realistic Cellular Automata Model to Simulate Traffic Flow at Urban Roundabouts
}

\author{
Ruili Wang and Mingzhe Liu \\ Institute of Information Sciences and Technology, Massey University, \\ Private Bag 11222, Palmerston North 5301, New Zealand \\ \{r.wang, m.z.liu\}@massey.ac.nz
}

\begin{abstract}
In this paper a realistic cellular automata model is proposed to simulate traffic flow at single-lane roundabouts. The proposed model is built on fine grid Cellular Automata (CA), so it is able to simulate actual traffic flow more realistically. Several important novel features are employed in our model. Firstly, 1.5-second rule is used for the headway (=distance /speed) in carfollowing process. Secondly, vehicles movement on urban streets are simulated based on the assumption of speed changes following a Gaussian (normal) distribution and is calibrated with the field data. Thirdly, driver behavior is modeled by using a truncated Gaussian distribution. Numerical results show that our method is feasible and valid.
\end{abstract}

\section{Introduction}

Roundabouts, regarded as complex subsystems, are important components of complex urban networks. The most important control rule in roundabouts is yield-at-entry [1], i.e. vehicles from the secondary roads give way to the vehicles on the circulatory road. Both empirical and theoretical methods [1] have been proposed to measure roundabouts performance such as capacity, delay, queue length, etc. With regard to these methods, the gap-acceptance criteria such as in [2, 3] are commonly used. Gapacceptance models are, however, unrealistic in general assuming that drivers are consistent and homogenous [4]. A consistent driver would be expected to behave in the same way in all similar situations, while in a homogenous population, all drivers have the same critical gap (the minimum time interval between two major-stream vehicles required by one minor-stream vehicle to pass through) and are expected to behave uniformly. The limitations of gap-acceptance models have been analyzed and detailed in literature [5]. Thus, in this paper we focus on using a Cellular Automata (CA) model to simulate traffic flow at an urban roundabout.

The employment of CA modeling traffic flow at roundabouts has attracted attention in the last few years [4-9], due to its dynamical and discrete characteristics [10] and its connection with stochasticity [11]. For a roundabout, vehicle maneuvers may include driving on the roads and on the roundabout.

Vehicles moving on the roads can be seen as driving on a straight urban road. Many models, such as in Ref. [7, 12, 13] have been developed to deal with driving on urban networks. To our knowledge, previous models normally implicitly assume that 
the headways (=distance/speed) are 1 second, that is, 2-second rule is not considered in those models. Theoretically, it should be observed by all drivers, although the headways that drivers use are shorter than 2 seconds [14] and normally longer than 1 second in the real world. In our research we have recorded 10 hours of traffic data between 16 August 2004 and 27 August 2004. The average car-following headway of 1.5 seconds has been observed in local urban networks and this 1.5 -second rule has been built into our model.

Wang and Ruskin [5] proposed a Minimal Acceptable sPace (MAP) method to simulate interactions between drivers at single-lane roundabouts. The method is able to simulate heterogeneous driver behavior and inconsistent driver behavior. In their model, driver behavior is randomly classified into four categories: conservative, rational, urgent and reckless, and each group has its own MAP. Meanwhile, inconsistent driver behavior is simulated by reassignment of categories with given probabilities at each time step. Although the assumption to categorize driver behavior into four groups is coarse, this approach, as far as we know, is the first model to reveal the impact of driver behavior on traffic flow at roundabouts.

Literature [7] proposed a stochastic CA interaction model. With this model, a waiting vehicle can enter the roundabout only if there are no vehicles on the roundabout in its left side quadrant. Obviously, this assumption is questionable. Each time step in the model is equivalent to 2 seconds. Clearly, the model is not able to describe traffic flow in details, such as acceleration or deceleration. A time step in micro-simulation is recommended between 0.1 and 1 second [2].

A simpler entry rule is also presented in [6], that is, if the cell located in front of the entrance is not occupied by the vehicle, a waiting vehicle is randomly generated and the cell is occupied. However, the yield-at-entry rule is not abided by and the speed of the following one on the circulatory lane is not considered, therefore, their model is unlikely to be a safety model.

We proposed a Normal Acceptable Space (NAS) model in this paper to describe heterogeneous driver behavior under normal conditions. The value of NAS is the number of required cells on a circulatory lane for a vehicle from a secondary road to enter the roundabout. The deviation of the NAS is used to model inconsistent driver behavior.

This paper is organized as follows. In Section 2, several important novel features are employed. Firstly, the average headway of 1.5 seconds is built into our model. Secondly, driver behavior and vehicle movement are modeled using a (truncated) Gaussian distribution. In this way, we present interaction rules at roundabouts according to the left-side driving such as in UK, Australia, and New Zealand. In Section 3, vehicle movement on urban roads is calibrated by field data and interaction models are also calibrated using field data provided in [19]. Furthermore, a comparison with other models is given and the result shows that our model is approximately consistent with other models. The conclusion is given in Section 4.

\section{Model Formulations}

In this paper a shorter length of cells is used in our model. In other words, the finer discretization of cells in our CA model is used comparing with previous models. The 
length of each cell is equal to $1 \mathrm{~m}$ in a real road, which provides a better resolution modeling actual traffic flow than other models. A unit of speed is therefore equal to $3.6 \mathrm{~km} / \mathrm{h}$ and each time step is 1 second. Since 1 unit of acceleration is $1 \mathrm{~m} / \mathrm{s}^{2}$, this also corresponds to a 'comfortable acceleration' [15].

In urban networks, a lower speed should be considered due to speed constraints. Normally, the legal limit of speed in urban networks is $50 \mathrm{~km} / \mathrm{h}$, however some people will drive at speeds about $58 \mathrm{~km} / \mathrm{h}$, which is just below the limit $(61 \mathrm{~km} / \mathrm{h})$ of being apprehended. Therefore, in our model, we assume the maximum speed of each vehicle is in the range of $50.4 \mathrm{~km} / \mathrm{h}-57.6 \mathrm{~km} / \mathrm{h}$. The speed corresponds to the number of cells, which a vehicle can move forward to in 1 second. The number of cells is 1416 cells.

Different vehicle types have different numbers of cells in lengths. Following are average values based on 10-hour recording data sets at morning peak hour and these are adopted in this paper.

Table 1. Vehicle components and required cells

\begin{tabular}{|l|c|c|}
\hline Vehicle Types & Occupied Cells & Percentage (\%) \\
\hline Motorcycles (M) & 3 & 2 \\
\hline Personal Vehicles (P) & 5 & 78 \\
\hline Vans and minibuses (V) & 7 & 11 \\
\hline Buses (B) & 10 & 6 \\
\hline Other large vehicles (O) & 13 & 3 \\
\hline
\end{tabular}

\subsection{Modeling Driver Behavior Under Gaussian Distribution}

As mentioned above, driver behavior is inconsistent, namely, even under similar conditions a driver may behave differently with time. So a driver can accept a space which value is shorter than the NAS due to long waiting time or other urgent conditions. Sometimes, a driver needs a space, which value is larger than the NAS due to bad weather, night visibility or other factors.

Let $x_{\min }$ represent the number of minimum acceptable cells and $x_{\max }$ stand for the number of maximum acceptable cells for a driver to interact with other drivers. If $x>$ $x_{\text {max }}$, a vehicle surely enters the roundabout without delay, but there is no interaction with other drivers. The values less than $x_{\min }$ are rejected due to safety factors and the values larger than $x_{\text {max }}$ are not included in consideration as no interaction is needed (free flow). Therefore, the model can be viewed as a truncated Gaussian distribution [16], where the left and right parts have been cut off. Mathematically, the truncated Gaussian distribution can be written as follows:

$$
f(x)=\frac{1}{\sigma \sqrt{2 \pi}} e^{\frac{(x-\mu)^{2}}{2 \sigma^{2}}} \quad x_{\min } \leq x \leq x_{\max }
$$

where $\mu$ is the value of the NAS and $\sigma$ is the value of deviation of the NAS.

From the statistical point of view, every vehicle entering roundabouts can be viewed as an independent event. According to the joint distribution theorem [17], if 
driver $A$ follows Gaussian distribution $N\left(\mu_{1}, \sigma_{1}^{2}\right)$, driver $B$ follows $N\left(\mu_{2}, \sigma_{2}^{2}\right), \ldots \ldots$, driver $M$ follows $N\left(\mu_{m}, \sigma_{m}{ }^{2}\right)$, then for independent drivers $A, B, \ldots \ldots, M$, the joint distribution of driver $A, B, \ldots, M$ follows Gaussian distribution $N\left(\mu, \sigma^{2}\right)$, namely,

$$
A+B+\ldots \ldots+M \sim N\left(\mu, \sigma^{2}\right)
$$

Concerning the above assumption, driver behavior can be modeled using Gaussian distribution. As such the heterogeneous driver behavior and inconsistent driver behavior can be incorporated by NAS and deviations from it.

\subsection{Modeling Vehicles Movement on Urban Streets}

The attention was mainly focused into modeling two of three phase traffic flow [18], namely, free flow and synchronized flow. In free flow, a vehicle can drive at its desired speed. In synchronized flow, a vehicle has to follow the vehicle in front. In free flow, speed changes of all vehicles can be assumed to follow a Gaussian distribution. This assumption is based on the fact that the speed changes of an individual vehicle can be approximately seen as a Gaussian distribution, which is described below.

\subsubsection{Free Flow Phase}

For a vehicle driving between intersections, speed changes are illustrated in Fig. 1, where five stages are involved in our model. In stage A, acceleration of the vehicle is delayed due to physical reasons. In stage $\mathrm{B}$, acceleration of the vehicle increases the speed drastically and leads to the desired speed in stage $C$. In stage $C$, speeds randomly fluctuate within the comfortable acceleration/deceleration range [15]. According to the distance between current position and the downstream junction, vehicles are slow down differently in stages $\mathrm{D}$ and $\mathrm{E}$. If the vehicle is following a vehicle in front, drivers adjust their speed depending upon speed changes of the preceding vehicle. Speed is adjusted as illustrated in Fig. 1. Probability density of each part follows Gaussian distribution. Update rules of the $n$th vehicle depends on its speed $v_{n}(t)$ at time step $t$ :

1. Speed adjustment

A: $v_{n}(t) \rightarrow v_{n}(t)+1$ B: $v_{n}(t) \rightarrow v_{n}(t)+2$

C: $v_{n}(t+1) \rightarrow v_{n}(t)-1$ with probability $p_{1}$ or, $v_{n}(t+1) \rightarrow v_{n}(t)+1$ with probability $p_{2}$ or, unchanged with probability $p_{3}$

$\mathrm{D}: v_{n}(t) \rightarrow v_{n}(t)-2$ E: $v_{n}(t) \rightarrow v_{n}(t)-1$

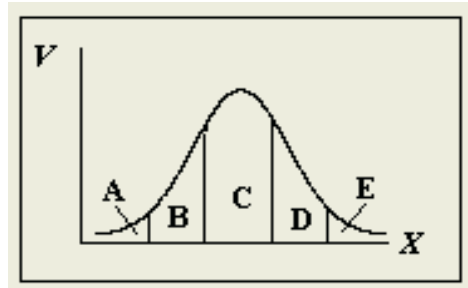

Fig. 1. Speed changes of vehicles in terms of the current position and the distance to the downstream junction. $\mathrm{V}$ and $\mathrm{X}$ denote the current speed and position, respectively

\subsubsection{Synchronized Flow Phase}

In synchronized flow, a vehicle has to follow the vehicle in front. Update rules of the $n$th vehicle depends on its position $x_{n}(t)$, speed $v_{n}(t)$, and gap (free cells in front) $g_{n}(t)$ at time step $t$ :

1. Speed adjustment

If $g_{n}(t)<v_{n}(t)$ then: $v_{n}(t+1) \rightarrow g_{n}(t+2 / 3)$ 
This rule is based on the 1.5 -second rule. In other words, the vehicle can only drive up to $2 / 3$ of the total distance between the vehicle and the vehicle in front.

Both in free and synchronized flow, the following steps are also implemented to simulate an overall vehicle movement.

2. Randomization

If $v_{n}(t)>0$, then the speed of the $n$-th vehicle is decelerated randomly with probability $p_{b}$, i.e. $v_{n}(t+1) \rightarrow \max \left\{0, v_{n}(t)-1\right\}$

3. Vehicle movement

$$
x_{n}(t+1) \rightarrow x_{n}(t)+v_{n}(t+1)
$$

Roundabouts are commonly used in where traffic is not heavy. In other words, the traffic flow that approach a roundabout are normally either free flow or synchronized flow, except at the entrance of a roundabout where queues may form. On the roundabout, the flow can be seen as synchronized flow.

\subsection{Modeling Interactions for Vehicles Entry Roundabouts}

Vehicles are numbered in the circulatory lane, namely, vehicle $n+1$ precedes vehicle $n$. Conditions for vehicle $k$ to enter the roundabout are described here. Vehicle $n$ and $n$ +1 are located on the roundabout, while vehicle $n+1$ has passed the entrance and vehicle $n$ is approaching the entrance. The vehicle $k$ is at the entrance and is waiting for entering the roundabout. Let $l_{k}$ denote the length of vehicle $k, m_{k}(t)$ denote NAS of vehicle $k, s_{k, n}(t)$ denote spacing between vehicle $k$ and $n$ at time $t$. Fig. 2 illustrates the location of vehicles and the topology of the road, and the roundabout. Concerning the above considerations, the following update rules are performed in parallel for all simulated vehicles:

1. Assigning NAS and its deviation for vehicle $k$ according to the probability density of Gaussian distribution.

2. Calculating $s_{k, n}(t)$. If $m_{k}(t) \leq s_{k, n}(t)$ and $l_{k} \leq s_{k, n+1}(t)$, the waiting vehicle $k$ can enter the roundabout or if $l_{k} \leq s_{k, n+1}(t)$, vehicle $k$ can also enter the roundabout, otherwise vehicle $k$ could not enter the roundabout.

3. If vehicle $k$ is waiting for entry, the update rule at each time step is as follows: $m_{k}(t)=m_{k}(t)-\sigma_{k}$ if a generated random number $\mathrm{R}(0 \leq \mathrm{R} \leq 1)<p, p$ is the predefined number within $[0,1]$, otherwise $m_{k}(t)=m_{k}(t)+\sigma_{k}$, where $m_{k}(t)$ and $\sigma_{k}$ are NAS (mean) and its deviation of vehicle $k$.

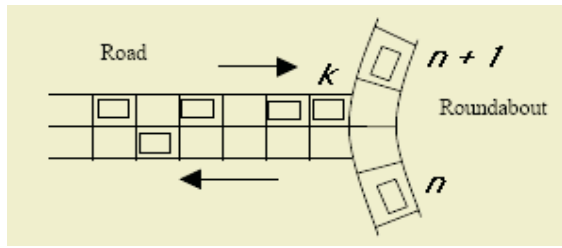

Fig. 2. Schematic diagram of vehicles distribution, a road and a part of the roundabout 


\section{Experimental Results}

The preliminary work is to calibrate vehicle movement on a straight lane. Fig. 3 shows observed single-vehicle movement and its simulation by using the proposed method. We found that when $p_{1}=p_{2}=0.3$ and $p_{3}=0.4$, the dual-regime of acceleration and deceleration of our simulation results fits the real behavior of vehicles well, especially in the initial acceleration and final deceleration phases. Probability density of each stage (see in Section 2.2) is assumed to follow Gaussian distribution.

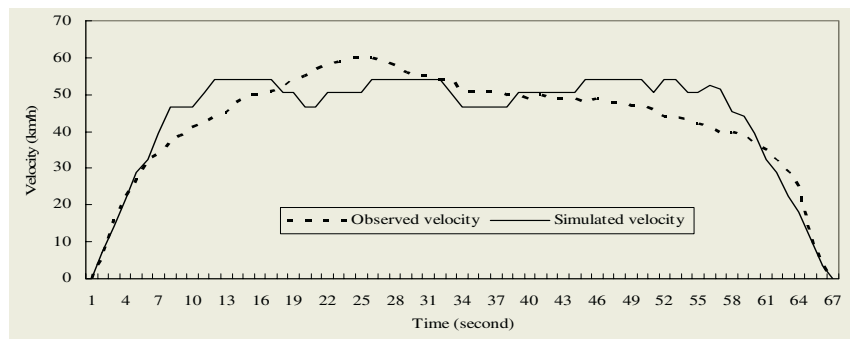

Fig. 3. Simulation of single-vehicle speed between two intersections

Next we apply our model to a case study. Experiments were implemented for 36000 time steps (equivalent to 10 hour) for a street-length of 100 cells on all approaches. The NAS of all drivers ranges within $\left[x_{\min }, x_{\max }\right]$, where $x_{\min }, x_{\max }$ are taken as 16 and 26 cells in terms of field observation. The mean and deviation of the truncated normal distribution are assumed to be 20 and 2 cells.

To carry out a realistic simulation, many input parameters are required, such as vehicles components, occupied cells, turning rate, arrival rate, etc. We use the data provided in [19] to verify our CA model, where vehicles types and component are given. Table 2 shows comparisons of capacity, delay, and queue length. We can find that capacity has an increase in our model, correspondingly, delay and queue length decrease slightly. For further verifying our model, a comparison with other models (aaSIDRA, UK Linear Regression, HCM 2000, NAASRA 1986) is given in Fig. 4, where capacity of roundabouts computed using our CA model is basically consistent with other models.

Table 2. Comparison capacity, delay, and 95\% queue length with our model and [19]. LT=left turning, ST=straight ahead, RT=right turning. Arm 1, 2, 3 and 4 are four roads connection with the roundabout

\begin{tabular}{|c|c|c|c|c|c|c|c|c|c|c|}
\hline \multirow[b]{2}{*}{ Road } & \multicolumn{3}{|c|}{ Vehicles Turning } & \multirow{2}{*}{$\begin{array}{c}\text { Vol } \\
\text {. }\end{array}$} & \multicolumn{2}{|c|}{ Capacity } & \multicolumn{2}{|c|}{ Delay } & \multicolumn{2}{|c|}{ 95\%Queue length } \\
\hline & LT & ST & RT & & [19] & Our model & [19] & Our model & [19] & Our model \\
\hline Arm 1 & 118 & 377 & 150 & 645 & 762 & 775 & 25 & 23 & 10 & 9.4 \\
\hline Arm 2 & 88 & 454 & 100 & 642 & 865 & 880 & 15 & 14 & 6.86 & 6.63 \\
\hline Arm 3 & 107 & 258 & 54 & 419 & 840 & 848 & 8.4 & 8.2 & 2.85 & 2.74 \\
\hline Arm 4 & 133 & 586 & 78 & 797 & 963 & 971 & 18.9 & 18 & 9.8 & 9.6 \\
\hline
\end{tabular}




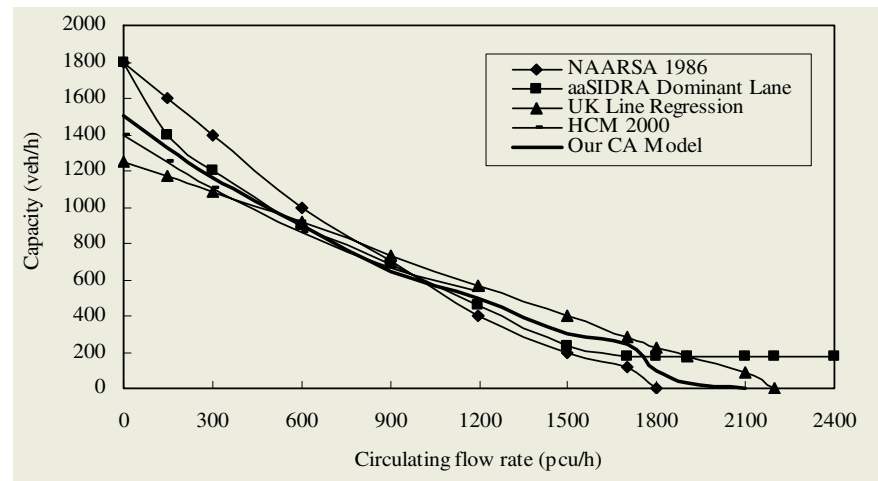

Fig. 4. Comparison of entry capacities estimated by our CA model and other models (the aaSIDRA, TRL (UK) Linear Regression, HCM 2000, NAASRA 1986) [1]

\section{Summary}

In this paper, we propose a realistic CA model to simulate traffic flow at an urban roundabout. Several important novel features are employed in our model. Firstly, it has been observed that the average headway of car-following is 1.5 seconds in local urban networks and this 1.5-second rule has been used in modeling the car-following process. Secondly, vehicle movement along urban streets is simulated based on the assumption that speed changes follow a Gaussian distribution. Thirdly, heterogeneous driver behavior and inconsistent driver behavior are modeled using the truncated Gaussian distribution.

Vehicle maneuver on urban roads has been calibrated using field data. The simulation results show that the dual-regime of acceleration and deceleration of the model fits with the real world well.

In order to model a realistic simulation, vehicle arrival rates, turning rates, vehicle types, driver behavior and categorization of speed, etc. are built into our model. The numerical results indicate that the performance (delay and queue length) of roundabouts can be described well.

\section{Acknowledgement}

The support of the Massey University Research Fund and the ASIA 2000 Foundation High Education Exchange Programme (HEEP) is a gratefully acknowledged.

\section{References}

1. Akçelik, R.: A Roundabout Case Study Comparing Capacity Estimates from Alternative Analytical Models. The $2^{\text {nd }}$ Urban Street Symposium, California, USA, 28-30 July 2003 
2. Flannery, A., Datta, T.: Operational performance measures of American roundabouts. Transportation research Record, 1572 (1997) 68-75.

3. Troutbeck, R.J.: Background for HCM section on analysis of performance of roundabouts. Transportation research Record, 1646 (1998) 54-62.

4. Wang, R.: Modelling Unsignalised traffic Flow with Reference to Urban and Interurban Networks. Doctorate Thesis. Dublin City University (2003)

5. Wang, R., and Ruskin, H.J.: Modeling Traffic Flow at a Single-lane Urban Roundabout, Computer Physics Communications, Vol. 147/1-2 (2002) 570-576, Elsevier Science.

6. Campari, E.G., Levi, G., Maniezzo, V.: Cellular automata and roundabout traffic simulation. Proceedings of ACRI 2004: Sixth International Conference on Cellular Automata for Research and Industry, Amsterdam, Netherland, 25-27 October 2004

7. Fouladvand, M.E., Sadjadi, Z. and Shaebani, M.R.: Characteristics of Vehicular Traffic Flow at a Roundabout. Preprints. cond-mat/0309560 (2003)

8. Wang, R., Ruskin, H.J.: Modelling Traffic Flow at a two-lane Roundabout, In: Proceedings of International Conference on Computer Science, Software Engineering, Information Technology, e-Business and Applications, June 5-7, 2003, Rio de Janeiro, Brazil.

9. Chopard, B., Dupuis, A. and Luthi, P.: Traffic and Granular Flow'97, World Scientific (1998) 153-168.

10. Toffoli, T., Margolus, N.: Cellular Automata Machines--A New Environment for Modelling http://pm1.bu.edu/ tt/cambook, MIT Press (1987)

11. Nagel, K., Schreckenberg, M.: A cellular automaton model for freeway traffic. J. Phys. I (France) 2 (1992) 2221-2229

12. Simon, P.M., Nagel, K.: Simplified cellular automata model for city traffic. Physical Review E Vol 58, (1998)

13. Barlovic, R., Brockfeld, E., Schreckenberg, M., Schadschneider, A.: Optimal traffic states in a cellular automaton model for city traffic. Traffic and Granular Flow, 2001.10.15 2001.10.17, Nagoya University, Japan

14. Neubert, L., Santen, L., Schadschneider, A. and Schreckenberg, M.: Single-vehicle data of highway traffic: A statistical analysis. Phys. Rev. E 60 (1999) 6480.

15. Institute of Transportation Engineers. Traffic Engineering Handbook. (1992)

16. Hays, W.L.: Statistics, the $5^{\text {th }}$ Edition, University of Texas as Austin, Harcourt Brace College Publishers (1994)

17. Kimber, R.M.: The Traffic Capacity of Roundabouts. TRRL Laboratory Report 942. Transportation and Road research Laboratory, Crowthorne, Berkshire, UK (1980)

18. Kerner, B.S. and Rehborn, H.: Experimental Properties of Phase Transitions in Traffic Flow. Phys. Rev. Lett. 79, (1997) 4030-4033

19. http://www.rpi.edu/dept/cits/files/ops.ppt, accessed on 12 October 2004 